\title{
Habib Zaidi (ed): Molecular Imaging of Small Animals: Instrumentation and Applications
}

\author{
Springer Science + Business Media, New York, 2014, ISBN 978-1-4939-0893-6
}

\author{
Giuseppe Danilo Di Stasio • Luigi Mansi
}

Published online: 28 January 2015

(C) Springer-Verlag Berlin Heidelberg 2015

The editor of this book is Habib Zaidi, Chief Physicist and Head of the PET Instrumentation and Neuroimaging Laboratory at Geneva University Hospital, and also a faculty member at the Medical School of Geneva University. In this volume, entitled Molecular Imaging of Small Animals: Instrumentation and Applications, Zaidi, in collaboration with international experts in the field, examines the most important techniques used for small-animal molecular imaging, analysing every aspect of each modality. All issues are covered starting from physics and ending with possible applications in preclinical research and future perspectives in humans. Throughout the book it is clear how important the development of small-animal imaging is to the authors, in enabling a better understanding of how the human body works at the molecular level.

In 760 pages, divided into 25 chapters and enriched by 289 illustration, of which 157 are in colour, is condensed all the authors' wisdom and knowledge on the subject. The method by which this large amount of information is organized is rational and strives to avoid redundancy among chapters. It fosters the use of figures to clarify concepts and to provide numerous molecular imaging examples. The first chapters focus on instrumentation: different types of detectors (including scintillation, solid-state, photon, MRI and CT detectors), single-modality systems such as SPECT, PET, CT, MRI and optical imaging, and dualmodality systems, including not only those already used in clinical practice in humans, such as PET/CT, SPECT/ $\mathrm{CT}$ and, more recently, PET/MRI, but also tools such as PET/SPECT, SPECT/MRI and PET/optical imaging. For

G. D. Di Stasio $\cdot$ L. Mansi $(\bowtie)$

Second University of Naples, Naples, Italy

e-mail: luigi.mansi@unina2.it all of these systems both design and physics and possible preclinical applications are discussed. The second half of the book highlights the possibilities that these kinds of studies give, with the main emphasis on in vivo quantitative assessment of metabolic and physiological parameters. Considering the similarities between small-animal models and human diseases, it is possible to understand how this analysis is of primary importance in many fields of study that are particularly well discussed in the book. An example is in cardiology, in which recent advances in imaging technologies and tracer development have opened the way for progress in studies of lipid accumulation in atherosclerosis and in remodelling after myocardial infarction. Oncology too benefits from molecular imaging techniques even more than other fields, due to the physiological and genetic alterations associated with cancer that can be utilized as targets either to create molecular probes for detecting neoplasms or to design new therapeutic tools. The last field of application analysed is drug development, in which small-animal molecular imaging contributes greatly to achieving a reduction in the time and cost required for modern drug discovery and the development process.

Such a state-of-the-art book, which reviews all imaging modalities in such a complete manner, clarifying both design principles and preclinical applications in biomedical research, has to be highly regarded whenever these matters are discussed by students or experts. In our opinion this publication should be available in all departments of nuclear medicine and diagnostic imaging, considering that molecular imaging is already living in the present. A deeper and wider knowledge in this field may significantly contribute not only to hypothesizing about the future, but also to better understanding and working in the present. 